\title{
The Next 50 Years of Neuroscience
}

\author{
Cara M. Altimus, ${ }^{1 \star}$ Bianca Jones Marlin, ${ }^{2 \star}$ Naomi Ekavi Charalambakis, ${ }^{3}$ Alexandra Colón-Rodríguez, ${ }^{4}$ \\ Elizabeth J. Glover, ${ }^{5}{ }^{\oplus}$ Patricia Izbicki, ${ }^{6}{ }^{\oplus}$ Anthony Johnson, ${ }^{7}{ }^{\oplus}$ Mychael V. Lourenco, ${ }^{8}$ Ryan A. Makinson, ${ }^{9}$ \\ Joseph McQuail, ${ }^{10}{ }^{\odot I g n a c i o ~ O b e s o, ~}{ }^{11}$ Nancy Padilla-Coreano, ${ }^{12}$ and ${ }^{\odot}$ Michael F. Wells, ${ }^{13}$ for Training Advisory Committee \\ ${ }^{1}$ Milken Institute, ${ }^{2}$ Columbia University Zuckerman Institute, ${ }^{3}$ University of Louisville, ${ }^{4}$ University of California at Davis, ${ }^{5}$ University of Illinois at Chicago, \\ ${ }^{6}$ Iowa State University, ${ }^{7}$ Veterans Administration Health Care System, ${ }^{8}$ Federal University of Rio do Janeiro, ${ }^{9}$ Journal of DoD Research \& Engineering, \\ ${ }^{10}$ University of South Carolina School of Medicine, ${ }^{11}$ Fundación de Investigación HM Hospitales, ${ }^{12}$ Salk Institute, and ${ }^{13}$ Broad Institute
}

On the 50th anniversary of the Society for Neuroscience, we reflect on the remarkable progress that the field has made in understanding the nervous system, and look forward to the contributions of the next 50 years. We predict a substantial acceleration of our understanding of the nervous system that will drive the development of new therapeutic strategies to treat diseases over the course of the next five decades. We also see neuroscience at the nexus of many societal topics beyond medicine, including education, consumerism, and the justice system. In combination, advances made by basic, translational, and clinical neuroscience research in the next 50 years have great potential for lasting improvements in human health, the economy, and society.

\section{Introduction}

In 1969, the United States National Academies Committee on Brain Sciences agreed that a central organization was needed to “1) advance understanding of nervous systems and their role in behavior; 2) promote education in the neurosciences; and 3) inform the general public on results and implications of current research." Thus, the Society for Neuroscience (SfN) was founded with the goal of serving as that central organization by bringing together neuroscientists across disciplines. Over the course of the last 50 years, members of the Society have been instrumental in driving the incredible growth and rapid technological advances that have accelerated our understanding of both healthy and pathological nervous system function.

As members of SfN's Trainee Advisory Committee, many of us joined the field within the last decade and recognize that it is our cohort's vision and drive that will advance the field through the next 50 years. Our Committee represents the vastness of neuroscience research, with members spanning broad scientific interests, ranging from neurodevelopment to neural correlates of behavior, and coming from countries around the world. As a diverse group of leaders within the Society, we think deeply about the next generation of neuroscientists and what their scientific world will look like because it is also what our world will look like. This article and timeline figure convey not only our vision of

\footnotetext{
Received April 1, 2019; revised Dec. 3, 2019; accepted Dec. 3, 2019.

The Trainee Advisory Committee is a group of current and recent trainees (graduate students and postdoctoral fellows) charged with making recommendations for the Society of Neuroscience Council and committees on how Society initiatives, projects, and programming can be designed, enhanced, or modified to address the needs and concerns of the Society's trainee members. All authors participated in this perspective as members of the 2019 Society for Neuroscience, Trainee Advisory Committee.

The authors declare no competing financial interests.

${ }^{*}$ C.M.A. and B.J.M. contributed equally to this work as chair and incoming chair, respectively, of the Trainee Advisory Committee.

Correspondence should be addressed to Bianca Jones Marlin at bjm2174@columbia.edu.

https://doi.org/10.1523/JNEUROSCI.0744-19.2019

Copyright $\odot 2020$ the authors
}

where the field will be, but are also a reflection of the research achievements that have driven us scientifically, and what we are most excited to see develop in the future (Extended Data Figure 1-1, available at https://doi.org/10.1523/JNEUROSCI.0744-19. 2019.f1-1). It is our hope that this vision will spur enthusiasm among professionals in the field and increase public understanding of the remarkable potential that neuroscience research holds to improve human health and society.

\section{Cellular and molecular neuroscience}

The past 50 years produced monumental advancements in our understanding of the cellular and molecular processes that dictate our every thought, desire, and action. This progress was driven, in part, by technical innovations, such as patch-clamp electrophysiology, PCR, and genomic sequencing, which gifted neuroscientists with experimental opportunities that were inconceivable in the 1960s. By the time SfN celebrates its 100th anniversary, we anticipate even greater shifts in methodology and conceptual consensus that will push the field further toward answering such questions as follows: How do the billions of individual components of the brain work together to generate behavior? How do changes to the brain lead to disease? What makes the human brain unique?

Two notable accomplishments toward answering these questions will be the completion of the connectome and a comprehensive cellular atlas of the mammalian brain. Execution of these daunting tasks is fueled in part by funding from National Institutes of Health's BRAIN Initiative, a 10 year program initiated in 2016 in the United States aiming to support the development and implementation of innovative neurotechnologies to better understand the brain (Bargmann, 2014), as well as the Human Brain Project funded by the European Union to foster research at the interface of neuroscience and computation and the Brain/ MINDS project in Japan focused on mapping higher brain function in marmosets. Among these new technologies are ongoing advancements in single-cell transcriptomics/proteomics, which 
will play a pivotal role in revealing the immense diversity of cell types within brains across a wide range of species (Saunders et al., 2018; Wang et al., 2018). In combination with the development of automated high-throughput and innovative optical electrophysiological approaches (Priest et al., 2004; Zhang et al., 2016), neuroscientists will begin to understand how discrete cell populations are physiologically and phylogenetically distinct. In doing so, we will determine not only the roles of specific cell types in the healthy and diseased brain, but also the cellular mechanisms that separate humans from other mammalian species. The data obtained will be used in conjunction with recently developed approaches, such as optogenetics (Boyden, 2011) and chemogenetics (Sternson and Roth, 2014), as well as new methods for visualizing genetically encoded calcium indicators (Resendez and Stuber, 2015), to revolutionize the ways in which we probe, perturb, and define distinct cell populations.

A cell's molecular makeup is vast and diverse, but neuroscientists' ability to resolve disease-induced alterations in molecular composition is currently laborious and imprecise. In the next 50 years, advances in microscopy (Gao et al., 2019) will become broadly accessible and afford researchers the ability to visualize subcellular machinery with unprecedented resolution, catapulting our understanding of the interplay between changes at the transcriptional, molecular, and structural levels. The development of new tools facilitating in vivo measurement and manipulation of epigenetic and molecular endpoints will revolutionize our ability to reconcile the influence of changes to the epigenome, genome, transcriptome, and proteome with behavior (HayashiTakagi et al., 2015). In vivo findings will be supplemented by studies performed in stem cell-derived cerebral organoids, which serve as a model of the developing human brain, and when combined with novel molecular and imaging tools, will begin to decipher the roles of specific cellular types and processes in the earliest stages of human neurodevelopment. Research over the next 50 years will further our understanding of the maturation of the synapse and the ways in which this critically important structure is regulated by complex signaling pathways, plasticity mechanisms, and non-neuronal elements, such as astrocytes, microglia, and the extracellular matrix (Dityatev et al., 2010; Prinz and Priller, 2014; Fields, 2015; Ben Haim and Rowitch, 2017).

Although initially these advancements will take a reductive approach, they will serve as a foundation upon which circuit and systems neuroscientists can build to gain a more thorough understanding of the brain across development, environment, and genetic background. Application of many of these tools in humans will be dependent on novel cellular targeting strategies that will make it easier for modified RNA, viral vectors, or small compounds to be directed to the cell types of choice, resulting in more accurate circuit manipulations and delivery of gene therapies and pharmaceuticals. These advances, combined with refined biomarkers of brain health, have the potential to vastly enhance our understanding of brain disease and open new avenues of therapeutic intervention.

\section{Development}

Building upon the advances in cellular and molecular neuroscience, the field of developmental neuroscience will be enabled to describe how internal and external factors shift the trajectory of individual neurons, circuits, and the brain to alter disease risk and behavior. Neurodevelopment spans intracellular study through systemwide analysis to allow an understanding of how individual neurons acquire specific function within the nervous system as well as how the brain develops over decades. While many avenues of research will be impactful, we see single-cell characterization, study of neurogenesis, and the use of organoids as key areas of focus in the next half century.

In particular, transcriptional characterization of neurons will be instrumental in providing a foundation by which researchers can study how cell fate, migratory paths, and connectivity are determined in unique cell types. Additionally, the use of whole genome sequencing to map cell lineage via the identification of somatic mutations (Evrony et al., 2015; Lodato et al., 2015) will provide crucial insight into the similarities and differences in cell dispersal in humans relative to other species. Together with broad implementation of new techniques that build upon the development of the Brainbow mouse by selectively labeling neurons undergoing differentiation or division (Gomez-Nicola et al., 2014; Loulier et al., 2014), these approaches will prove essential to allow neuroscientists to monitor the fate of individual neural progenitors and their trajectories as they form the complex circuits that define the nervous system.

The past 50 years of neuroscience research has played host to a decades-long debate over the existence of adult neurogenesis. This controversial concept was first introduced before SfN's formation in 1969 (Altman, 1962) but failed to gain significant traction until the 1980s and 1990s when an increasing number of reports demonstrated the presence of newly born cells originating in the subventricular and subgranular zones of a number of species, including humans (Eriksson et al., 1998; Knoth et al., 2010; Spalding et al., 2013). Nevertheless, despite convincing evidence, the debate has continued, with recent work suggesting that adult hippocampal neurogenesis is minimal, if not absent, at least in primates (Sorrells et al., 2018). Yet, an even more recent study created additional controversy by demonstrating adult hippocampal neurogenesis is robust in healthy aged individuals (Boldrini et al., 2018; Moreno-Jiménez et al., 2019). The reasons for the continued controversy likely relate to technical limitations arising from the use of postmortem tissues with variable fixation protocols, possibly measuring the wrong marker(s) for neural stem cells, and from attempting to generalize results from rodent models. Over the course of the next five decades, we expect that new technologies to definitively label new neurons in vivo via noninvasive imaging techniques or in ex vivo samples across mammalian species will move the field forward. These continuing attempts to resolve this issue will lead to even deeper insights into complex mechanisms of cortical development in primates. Additionally, by applying results from 'omic studies of developing neurons, we anticipate that tools may be developed to precisely control neurogenesis to modify disease processes and understand its roles in physiology and disease.

Since their introduction in 2013, brain organoids have presented neuroscientists with a model system that can be used to study a myriad of processes, including brain development and aging (Lancaster et al., 2013; Gonzalez et al., 2018; Karzbrun and Reiner, 2019; Pollen et al., 2019). While protocols have been established to grow brain organoids from embryonic or induced pluripotent stem cells (Lancaster et al., 2013; Sloan et al., 2017; Karzbrun and Reiner, 2019), several methodological drawbacks have prevented them from realizing their full research potential (Karzbrun and Reiner, 2019; Yakoub, 2019; Yoon et al., 2019). Technological advances in the coming years will resolve the vascular and structural support difficulties researchers currently experience to allow the growth of larger, highly reproducible organoids that more closely resemble the complexity of the developing human brain. These advances will usher in a new era of 
in vitro research, enabling investigators to explore numerous facets of developmental neuroscience. In combination with live-cell imaging, brain organoids will vastly accelerate progress in understanding the complex signaling patterns that drive cell fate, neuronal migration, and neurite extension. Computational approaches, such as those developed for systems modeling, have thus far only seen limited application in developmental neuroscience; however, these methods will allow researchers to study the complex interplay between the seemingly infinite number of spatially and temporally distinct signals driving cell fate, neuronal migration, and circuit formation, which to date have largely been studied in isolation. Brain organoid experiments using viral strategies to measure and manipulate neuronal activity will also be pivotal in elucidating the role that experience-dependent plasticity plays in the formation and maintenance of neural circuits. Together with the development of more broadly accessible methods to manipulate cell structure in vivo (Hayashi-Takagi et al., 2015), these advances will allow neuroscientists to better understand the mechanisms underlying synapse formation and link structural plasticity with synaptic plasticity and behavior.

In addition to improving our understanding of the mechanisms underlying neural development, organoids will afford researchers a system for studying characteristics of the nervous system that are uniquely human and contribute to our knowledge of neurodevelopmental diseases, including autism and schizophrenia, which have proven difficult to study in animal models (Di Lullo and Kriegstein, 2017). With the ability to form functional circuits that could persist for months or potentially years, neuroscientists will be able to test the effects of genetics, age, and environment impact on brain function by comparing healthy cell lines to lines with genetic errors across time and in response to environmental stressors. Ultimately, brain organoids will become a standard model to screen pharmaceuticals and test the efficacy of gene editing techniques as therapies for neurological diseases. Furthermore, this technology may one day provide the means to correct damage resulting from injury or disease using self-derived replacement brain tissue.

\section{From systems to behaviors}

Historically, neuroscientists have taken a reductionist approach to understanding brain function. Our modern understanding of the brain has evolved over the past century, from the limited 47 brain regions known in 1909, to our current human brain map with 98 regions in the cortex, alone (Glasser et al., 2016). Initially, neuroscientists relied on lesions or pharmacological manipulations in animals to determine the role of a given brain region. However, within the past two decades, new genetic tools have increased our ability to precisely manipulate circuits in animal models with increased precision. Such studies have heightened our understanding of the circuits underlying sensory processing, motor control, and memory. Questions still remain, as much of the work to date has focused on examining these circuits in isolation, therefore making limited progress in understanding how multiple regions or circuits interact to produce a behavioral output. For example, how do circuits for motor control, sensory processing, and decision making interact? How do manipulations of sensory processing affect the computations of planned motor movement?

Given that several brain systems are well understood individually, and the field has developed techniques with higher temporal and spatial resolution to monitor and manipulate neural activity, we are now better positioned to start deciphering how groups of neurons and distant regions work together to drive behavior. For this, the use of high-density multisite electrode recordings in the full brain will transform the field. Additionally, virtual reality environments, model-based analyses, and artificial intelligence approaches can be combined with these new recording and manipulation techniques to allow researchers to study how multiple sensory inputs are integrated and transformed into a behavioral output (e.g., action, thought, decision). Zebrafish and Caenorhabditis elegans will also prove instrumental in studying how multiple functional circuits operate in tandem because these animals offer researchers an opportunity to image the activity of the entire nervous system at once in conjunction with behavioral monitoring (Cong et al., 2017). As neuroscientists sample more neurons with high-density electrodes or imaging methods, it will be important to attempt to understand what all the neurons are encoding, not just the neurons that are taskresponsive or that support the specific hypotheses in the study. For this effort, statistical and computational methods, such as machine learning, will become essential and open up whole new areas of neuroengineering.

The recent development of virally mediated gene-editing strategies to optically measure and manipulate selected groups of neurons in vivo has been a boon for systems neuroscientists. These new technologies have moved circuit-based experiments into the limelight and are rapidly elucidating the connections, and the specific role of unique neural populations. Over the next 50 years, these techniques will provide the foundation for monumental breakthroughs in our understanding of how neural ensembles guide behavior (Jennings et al., 2019), and perhaps even consciousness. Consciousness, in particular, is an important target of in-depth investigation as the very experience of awareness in ourselves and the world around us likely drives cognitive functioning (e.g., action planning or decision making) and may be modulated by diseases and conditions that affect the brain. Together with cellular-resolution functional human neuroimaging approaches, which are just beginning to be realized (Koopmans and Yacoub, 2019), cognitive neuroscientists will begin to unlock the still poorly understood complexity of the distinct brain regions, such as the cerebellum, PFC, or hippocampus as well as how activity in multiple regions work in concert with each other. For example, higher-resolution imaging of the human brain will allow deciphering new understanding of circuit functionalities paving the way for neural modulatory interventions, such as transcranial magnetic stimulation and ultrasound neuromodulation. These circuit-based intervention strategies could be used to treat neuropsychiatric illness by manipulating a functionally distinct neural hub (Diana et al., 2017).

In light of incredible strides, systems neuroscience has been limited by the way behavior is measured and correlated to neural activity. The ability of neuroscientists to resolve distinct functional circuits is limited by the precision with which behavior is defined and measured, often manually or semiautomatically by a human observer, resulting in oversimplified endpoints and frequently overlooked details (Anderson and Perona, 2014). Additionally, behavioral measurements are especially rudimentary for social behaviors in animals. Over the next 50 years, approaches used in behavioral neuroscience will more closely resemble the sophisticated methods being used to functionally dissect neural circuits. Computer vision technology will enable fully automated, high-throughput, unbiased behavioral analysis, exponentially pushing the field forward (Wiltschko et al., 2015; Mathis et al., 2018). Moreover, our ability to track behavior continuously and reliably in social settings will open the door to the development of new animal models of neuropsychiatric diseases, such as 
anxiety and depression, for which current models are overly simplistic. Similarly, approaches in humans (e.g., using in-home laboratories, online experiments, neurofeedback) (Awolusi et al., 2018; Marins et al., 2019) have the potential to uncover previously unrealized symptomology and/or behavioral indices of risk for disease (Anderson and Perona, 2014; Wiltschko et al., 2015; Cong et al., 2017; Mathis et al., 2018; Jennings et al., 2019).

Finally, through combining technologies to record and interact with neural circuits in real time, as well as incorporating unbiased methodologies to characterizing behavior and neural activity, we will see transformation on neural interface technologies that directly engage the nervous system. This technology is currently undergoing rapid advancement with brain-computer interfaces successfully allowing control of prosthetic limbs and perception of rudimentary visual imagery in the blind. As these technologies advance, there is hope that these neural interfaces will advance to allow broader application for prosthetic limbs, inclusion of sensory feedback, and perhaps memory improvement in individuals who experience cognitive decline.

\section{Disease}

Over the last 50 years, scientific discoveries have improved our understanding of how specific diseases disrupt nervous system function. Fortunately, we are moving past a time when individuals affected by conditions, such as autism, depression, schizophrenia, and dementia, are institutionalized, stigmatized, and marginalized. Today, policymakers and society rely heavily on neuroscientists to inform them about the role of the brain in these conditions and the advances in detection, prognosis, and treatment for patients affected by neurological and neuropsychiatric disorders. Over the next 50 years, we anticipate that disease research will address the following questions: What molecular/cellular changes happen in the brain before the onset of nervous system disorders? How can we harness the biological understanding of a disease to develop targeted therapeutics to tackle the full complexity and multifactorial nature of neurological disease? How can we intervene early to prevent disease manifestation and/or progression?

With this in mind, 50 years from now, we predict we will be celebrating an era of "neurotherapeutics." The beginnings of this era are already upon us, as an impressive number of neuro-based therapies have recently gained FDA approval: examples are esketamine for major depressive disorder, brexanolone for postpartum depression, and siponimod for multiple sclerosis (Urquhart, 2019). However, today drugs intended to treat nervous system disorders take longer and are less likely to gain FDA approval compared with other drugs (Gaffney, 2014). Similar to the strong progress made in cancer treatment over the last 30 years, an increase in the number of successful therapies for the treatment of nervous system disorders will be driven largely by public and political support for funding directed toward such endeavors. The BRAIN Initiative has already played a fundamental role in the development of technologies that likely will greatly impact disease diagnosis and treatment. This funding, in addition to disease-specific funding, such as US Department of Health \& Human Services' National Plan to Address AD and associated federal funding dedicated to $\mathrm{AD}$ research, as well as the dementia research initiatives led by the United Kingdom (Fox and Petersen, 2013), has the potential to lead to faster translation and compounded discovery for prioritized classes of neural-based illness.

Beyond therapeutic development, we will also apply biological and mechanistic understanding to the diagnosis of neurological and psychiatric conditions. Specifically, we will transition from a symptom-based approach to one that also considers etiological agents and molecular intricacies. This realignment is exemplified by the use of genotype to define spinal muscular atrophy, as well as a new research criteria in which molecular alterations in the brain are used to classify dementias, including $\mathrm{AD}$, even in the absence of clinical or postmortem neuropathology (Jack and Vemuri, 2018; Khachaturian et al., 2018). Improved sensitivity and multiplexing of blood tests and other minimally invasive tests to detect changes in brain function will aid in extending this approach to diseases other than spinal muscular atrophy and $\mathrm{AD}$. Technological advances, such as activity trackers and artificial intelligence, will have profound impacts on how we understand normal and abnormal function and treat neurological disorders. Artificial intelligence has already revealed specific plasma biomarker combinations to improve AD diagnostics (Ashton et al., 2019) and will be used similarly in the future to more efficiently analyze the efficacy of pharmacotherapies in larger biobanks, thereby expediting the discovery of new therapies. Additionally, the development of new tracers compatible with positron emission tomography imaging hold promise as a valuable diagnostic and prognostic resource (Leuzy et al., 2018).

Alongside the investment of time, resources, and effort in finding cures for brain illnesses, it will be imperative to foster research on preventive mechanisms. The high prevalence of neurological diseases worldwide is socially demanding and economically expensive. Therefore, defining the essential mechanisms by which tractable lifestyle interventions (physical exercise, diet, cognitive training, and engagement in social, cultural, and educational activities) could potentially modify disease risk should be an enduring research priority throughout the upcoming 50 years. Likewise, interrogation of genetic and environmental susceptibility factors (e.g., polymorphisms, exposure to toxins) may reveal important clues to inform health policies and medical practice in the future.

In total, we see the advancements in cellular, developmental, and systems neuroscience culminating in dramatic improvements for nervous system illnesses through improved understanding of underlying mechanisms of disease, identification of new diagnostic endpoints to detect disease before symptom onset, and ultimately, new methods for treatment and prevention.

\section{An inclusive future}

It is clear that the next 50 years will be marked not only by a more comprehensive understanding of the system that allows us to interact with the world around us, but also by fundamental changes in how neuroscience research is accomplished and the very topics that are studied. Among these changes, neuroscientists must acknowledge the importance of diversity. To date, research in male (across species) (Shansky, 2019) and right-handed subjects has predominated. Additionally, clinical trials and genetic studies continue to overwhelmingly assess individuals of European descent. These systemic barriers to a comprehensive understanding of neuroscience, and the individual differences contained therein, are driven, in part, by a lack of diversity within neuroscientists themselves. As a result, the field suffers from a lack of understanding with respect to sex differences and the female brain, and FDA- or EMA-approved drugs frequently exhibit decreased therapeutic efficacy in nonwhite populations. Looking forward, we must prioritize greater diversity in both our researchers and our research subjects. 


\section{Neuroscience in society}

The impacts of neuroscience research extend far beyond the clinic to the classroom, the courtroom, and even the grocery store. Indeed, neuro-technologies are already moving into our homes, promising to boost cognitive abilities, despite insufficient rigorous evidence of efficacy (Nelson et al., 2016; Schuijer et al., 2017).

Neuroeducation, a field that combines research findings in developmental and cognitive neuroscience with educational strategies (Sigman et al., 2014), has contributed greatly to our understanding of how students with dyslexia, attention deficit hyperactivity disorder, and other disorders learn. This knowledge has been used to implement changes in math, arts, and science curricula for students with these disorders. Recent evidence also shows that intertwining arts and science education allows students to find more creative and innovative approaches to solving problems. Despite this progress, cognitive psychology and neuroscience are not broadly implemented in standard educational practices of teachers in both primary and higher education (Sigman et al., 2014). Further application of neuroscience and development of research in this space are beginning to change when mathematics concepts are taught and fundamentally change the way we schedule school days to align with circadian rhythms. Over the course of the next 50 years, we expect to see broader application of neuroeducational strategies across age and educational setting.

Neuroscience is becoming increasingly more common in the courtroom as it is used to explain criminal behavior (Ward et al., 2018). Its use will increase over the next 50 years as researchers become more knowledgeable about the neurobiological mechanisms underlying decision making. Moreover, as diagnostic tools, human neuroimaging methods in particular, become more advanced and afford researchers greater insight into brain function, these strategies will be used to determine an individual's culpability and even likelihood for recidivism.

Although it may not be apparent in our everyday lives, companies all over the world are using the results of neuroscience research to inform their business practices from office structure to product placement and marketing strategies. This will likely increase over the course of the next five decades as our understanding of the neurobiology of cognition and attention matures (Gottlieb and Oudeyer, 2018). In particular, wearable neurotechnology has the potential to play a prominent role in providing instant consumer feedback allowing personalized marketing strategies that update in real-time (Awolusi et al., 2018). However, companies should exercise caution and follow ethical principles when developing new strategies to generate profit based on neurobiological understanding and techniques.

In conclusion, neuroscience is a vast field. With $\sim 86$ billion neurons in the adult human brain, and approximately the same number of non-neuronal cells, it is not surprising that the study of this organ is complex. Furthermore, the nervous system extends far beyond the cranium with neurons projecting to the furthest reaches of the body collecting input and responding to the environment. The progress the field continues to reinforces its enormous potential.

Beyond examining the complexity of the nervous system itself, we must ask ourselves how we study this system of systems. When considering the approach that other scientific fields with seemingly infinite complexity have taken, the study of space comes to mind. While individual nations have embarked on space exploration over the last century, collaboration across disciplines and countries likely contributed to the great strides made thus far.
Borrowing from this example, interdisciplinary approaches, with teams of mathematicians, engineers, computer scientists, biologists, and chemists, are key to the continued advancement of neuroscience. Presently, neuroscience is funded in many countries through numerous agencies; however, recent national and international initiatives facilitating large-scale interdisciplinary neuroscience are emerging. The BRAIN Initiative and the Human Brain Project, for example, have not focused on one specific area of neuroscience but instead embraced participation from researchers spanning science, engineering, math, and technology.

The vitality of SfN, whose annual meeting has grown from 1395 to $>30,000$ attendees per year, highlights its immense value as a central space for scientific dialog and collaboration (Fields, 2018). Expansion of these centrally coordinated efforts to accelerate brain research as well as a strong community of scientists will be instrumental in elevating the quality and capability of neuroscience research as it continues to explore the unknown.

\section{References}

Altman J (1962) Are new neurons formed in the brains of adult mammals? Science 135:1127-1128.

Anderson DJ, Perona P (2014) Toward a science of computational ethology. Neuron 84:18-31.

Ashton NJ, Alejo J, Nevado-Holgado AJ, Barber IS, Lynham S, Gupta V, Chatterjee P, Goozee K, Hone E, Pedrini S, Blennow K, Schöll M, Zetterberg H, Ellis KA, Bush AI, Rowe CC, Villemagne VL, Ames D, Masters CL, Aarsland D, Powell J, et al. (2019) A plasma protein classifier for predicting amyloid burden for preclinical Alzheimer's disease. Sci Adv 5:eaau7220.

Awolusi I, Marks E, Hallowell M (2018) Wearable technology for personalized construction safety monitoring and trending: review of applicable devices. Automation Construction 85:96-106.

Bargmann C (2014) BRAIN 2025: a scientific vision.

Ben Haim L, Rowitch DH (2017) Functional diversity of astrocytes in neural circuit regulation. Nat Rev Neurosci 18:31-41.

Boldrini M, Fulmore CA, Tartt AN, Simeon LR, Pavlova I, Poposka V, Rosoklija GB, Stankov A, Arango V, Dwork AJ, Hen R, Mann JJ (2018) Human hippocampal neurogenesis persists throughout aging. Cell Stem Cell 22: 589-599.e5.

Boyden ES (2011) A history of optogenetics: the development of tools for controlling brain circuits with light. F1000 Biol Rep 3:11.

Cong L, Wang Z, Chai Y, Hang W, Shang C, Yang W, Bai L, Du J, Wang K, Wen Q (2017) Rapid whole brain imaging of neural activity in freely behaving larval zebrafish (Danio rerio). Elife 6:e28158.

Diana M, Raij T, Melis M, Nummenmaa A, Leggio L, Bonci A (2017) Rehabilitating the addicted brain with transcranial magnetic stimulation. Nat Rev Neurosci 18:685-693.

Di Lullo E, Kriegstein AR (2017) The use of brain organoids to investigate neural development and disease. Nat Rev Neurosci 18:573-584.

Dubinsky JM (2010) Neuroscience education for prekindergarten-12 teachers. J Neurosci 30:8057-8060.

Eriksson PS, Perfilieva E, Björk-Eriksson T, Alborn AM, Nordborg C, Peterson DA, Gage FH (1998) Neurogenesis in the adult human hippocampus. Nat Med 4:1313-1317.

Evrony GD, Lee E, Mehta BK, Benjamini Y, Johnson RM, Cai X, Yang L, Haseley P, Lehmann HS, Park PJ, Walsh CA (2015) Cell lineage analysis in human brain using endogenous retroelements. Neuron 85:49-59.

Fields RD (2015) A new mechanism of nervous system plasticity: activitydependent myelination. Nat Rev Neurosci 16:756-767.

Fields RD (2018) The first Annual Meeting of the Society for Neuroscience, 1971: reflections approaching the 50th anniversary of the Society's formation. J Neurosci 38:9311-9317.

Fox NC, Petersen RC (2013) The G8 dementia research summit-a starter for eight? Lancet 382:1968-1969.

Gaffney A (2014) Report finds FDA slow to approve CNS drugs, but getting faster. November 5, 2014. https://www.raps.org/regulatory-focus\%E2\% 84\%A2/news-articles/2014/11/report-finds-fda-slow-to-approve-cnsdrugs,-but-getting-faster. 
Gao R, Asano SM, Upadhyayula S, Pisarev I, Milkie DE, Liu TL, Singh V, Graves A, Huynh GH, Zhao Y, Bogovic J, Colonell J, Ott CM, Zugates C, Tappan S, Rodriguez A, Mosaliganti KR, Sheu SH, Pasolli HA, Pang S, et al. (2019) Cortical column and whole-brain imaging with molecular contrast and nanoscale resolution. Science 363:eaau8302.

Glasser MF, Coalson TS, Robinson EC, Hacker CD, Harwell J, Yacoub E, Ugurbil K, Andersson J, Beckmann CF, Jenkinson M, Smith SM, Van Essen DC (2016) A multi-modal parcellation of human cerebral cortex. Nature 536:171-178.

Gomez-Nicola D, Riecken K, Fehse B, Perry VH (2014) In-vivo RGB marking and multicolour single-cell tracking in the adult brain. Sci Rep 4:7520.

Gonzalez C, Armijo E, Bravo-Alegria J, Becerra-Calixto A, Mays CE, Soto C (2018) Modeling amyloid beta and tau pathology in human cerebral organoids. Mol Psychiatry 23:2363-2374.

Gottlieb J, Oudeyer PY (2018) Towards a neuroscience of active sampling and curiosity. Nat Rev Neurosci 19:758-770.

Hayashi-Takagi A, Yagishita S, Nakamura M, Shirai F, Wu YI, Loshbaugh AL, Kuhlman B, Hahn KM, Kasai H (2015) Labelling and optical erasure of synaptic memory traces in the motor cortex. Nature 525:333-338.

Jack CR Jr, Vemuri P (2018) Amyloid- $\beta$ : a reflection of risk or a preclinical marker? Nat Rev Neurol 14:319-320.

Jennings JH, Kim CK, Marshel JH, Raffiee M, Ye L, Quirin S, Pak S, Ramakrishnan C, Deisseroth K (2019) Interacting neural ensembles in orbitofrontal cortex for social and feeding behaviour. Nature 565:645-649.

Karzbrun E, Reiner O (2019) Brain organoids: a bottom-up approach for studying human neurodevelopment. Bioengineering (Basel) 6:E9.

Khachaturian AS, Hayden KM, Mielke MM, Tang Y, Lutz MW, Gustafson DR, Kukull WA, Mohs R, Khachaturian ZS (2018) Future prospects and challenges for Alzheimer's disease drug development in the era of the NIA-AA research framework. Alzheimers Dement 14:532-534.

Knoth R, Singec I, Ditter M, Pantazis G, Capetian P, Meyer RP, Horvat V, Volk B, Kempermann G (2010) Murine features of neurogenesis in the human hippocampus across the lifespan from 0 to 100 years. PLoS One 5:e8809.

Koopmans PJ, Yacoub E (2019) Strategies and prospects for cortical depth dependent T2 and T2* weighted BOLD fMRI studies. Neuroimage 197:668-676.

Lancaster MA, Renner M, Martin CA, Wenzel D, Bicknell LS, Hurles ME, Homfray T, Penninger JM, Jackson AP, Knoblich JA (2013) Cerebral organoids model human brain development and microcephaly. Nature 501:373-379.

Leuzy A, Heurling K, Ashton NJ, Schöll M, Zimmer ER (2018) In vivo detection of Alzheimer's disease. Yale J Biol Med 91:291-300.

Lodato MA, Woodworth MB, Lee S, Evrony GD, Mehta BK, Karger A, Lee S, Chittenden TW, D'Gama AM, Cai X, Luquette LJ, Lee E, Park PJ, Walsh CA (2015) Somatic mutation in single human neurons tracks developmental and transcriptional history. Science 350:94-98.

Loulier K, Barry R, Mahou P, Le Franc Y, Supatto W, Matho KS, Ieng S, Fouquet S, Dupin E, Benosman R, Chédotal A, Beaurepaire E, Morin X, Livet J (2014) Multiplex cell and lineage tracking with combinatorial labels. Neuron 81:505-520.

Marins T, Rodrigues EC, Bortolini T, Melo B, Moll J, Tovar-Moll F (2019) Structural and functional connectivity changes in response to short-term neurofeedback training with motor imagery. Neuroimage 194:283-290.

Mathis A, Mamidanna P, Cury KM, Abe T, Murthy VN, Mathis MW, Bethge M (2018) DeepLabCut: markerless pose estimation of user-defined body parts with deep learning. Nat Neurosci 21:1281-1289.

Moreno-Jiménez EP, Flor-García M, Terreros-Roncal J, Rábano A, Cafini F, Pallas-Bazarra N, Ávila J, Llorens-Martín M (2019) Adult hippocampal neurogenesis is abundant in neurologically healthy subjects and drops sharply in patients with Alzheimer's disease. Nat Med 25:554-560.

Nelson J, McKinley RA, Phillips C, McIntire L, Goodyear C, Kreiner A, Monforton L (2016) The effects of transcranial direct current stimulation (TDCS) on multitasking throughput capacity. Front Hum Neurosci 10:589.

Pollen AA, Bhaduri A, Andrews MG, Nowakowski TJ, Meyerson OS,
Mostajo-Radji MA, Di Lullo E, Alvarado B, Bedolli M, Dougherty ML, Fiddes IT, Kronenberg ZN, Shuga J, Leyrat AA, West JA, Bershteyn M, Lowe CB, Pavlovic BJ, Salama SR, Haussler D, et al. (2019) Establishing cerebral organoids as models of human-specific brain evolution. Cell 176: 743-756.e17.

Priest BT, Cerne R, Krambis MJ, Schmalhofer WA, Wakulchik M, Wilenkin B, Burris KD (2004) Automated electrophysiology assays. In: Assay guidance manual (Sittampalam GS, Coussens NP, Brimacombe K, Grossman A, Arkin M, Auld D, Austin C, et al., eds). Bethesda, MD: Eli Lilly and the National Center for Advancing Translational Sciences.

Prinz M, Priller J (2014) Microglia and brain macrophages in the molecular age: from origin to neuropsychiatric disease. Nat Rev Neurosci 15: $300-312$.

Resendez SL, Stuber GD (2015) In vivo calcium imaging to illuminate neurocircuit activity dynamics underlying naturalistic behavior. Neuropsychopharmacology 40:238-239.

Saunders A, Macosko EZ, Wysoker A, Goldman M, Krienen FM, de Rivera H, Bien E, Baum M, Bortolin L, Wang S, Goeva A, Nemesh J, Kamitaki N, Brumbaugh S, Kulp D, McCarroll SA (2018) Molecular diversity and specializations among the cells of the adult mouse brain. Cell 174: 1015-1030.e16.

Schuijer JW, de Jong IM, Kupper F, van Atteveldt NM (2017) Transcranial electrical stimulation to enhance cognitive performance of healthy minors: a complex governance challenge. Front Hum Neurosci 11:142.

Shansky RM (2019) Are hormones a 'female problem' for animal research? Science 364:825-826.

Sigman M, Peña M, Goldin AP, Ribeiro S (2014) Neuroscience and education: prime time to build the bridge. Nat Neurosci 17:497-502.

Sloan SA, Darmanis S, Huber N, Khan TA, Birey F, Caneda C, Reimer R, Quake SR, Barres BA, Pasca SP (2017) Human astrocyte maturation captured in $3 \mathrm{D}$ cerebral cortical spheroids derived from pluripotent stem cells. Neuron 95:779-790.e6.

Sorrells SF, Paredes MF, Cebrian-Silla A, Sandoval K, Qi D, Kelley KW, James D, Mayer S, Chang J, Auguste KI, Chang EF, Gutierrez AJ, Kriegstein AR, Mathern GW, Oldham MC, Huang EJ, Garcia-Verdugo JM, Yang Z, Alvarez-Buylla A (2018) Human hippocampal neurogenesis drops sharply in children to undetectable levels in adults. Nature 555:377-381.

Spalding KL, Bergmann O, Alkass K, Bernard S, Salehpour M, Huttner HB, Boström E, Westerlund I, Vial C, Buchholz BA, Possnert G, Mash DC, Druid H, Frisén J (2013) Dynamics of hippocampal neurogenesis in adult humans. Cell 153:1219-1227.

Sternson SM, Roth BL (2014) Chemogenetic tools to interrogate brain functions. Annu Rev Neurosci 37:387-407.

Urquhart L (2019) FDA new drug approvals in Q1 2019. Nat Rev Drug Discov. Advance online publication. Retrieved Apr 10, 2019. doi: 10.1038/ d41573-019-00070-3.

Wang X, Allen WE, Wright MA, Sylwestrak EL, Samusik N, Vesuna S, Evans K, Liu C, Ramakrishnan C, Liu J, Nolan GP, Bava FA, Deisseroth K (2018) Three-dimensional intact-tissue sequencing of single-cell transcriptional states. Science 361:eaat5691.

Ward T, Wilshire C, Jackson L (2018) The contribution of neuroscience to forensic explanation. Psychol Crime Law 24:195-209.

Wiltschko AB, Johnson MJ, Iurilli G, Peterson RE, Katon JM, Pashkovski SL, Abraira VE, Adams RP, Datta SR (2015) Mapping sub-second structure in mouse behavior. Neuron 88:1121-1135.

Yakoub AM (2019) Cerebral organoids exhibit mature neurons and astrocytes and recapitulate electrophysiological activity of the human brain. Neural Regen Res 14:757-761.

Yoon SJ, Elahi LS, Pasca AM, Marton RM, Gordon A, Revah O, Miura Y, Walczak EM, Holdgate GM, Fan HC, Huguenard JR, Geschwind DH, Pasca SP (2019) Reliability of human cortical organoid generation. Nat Methods 16:75-78.

Zhang H, Reichert E, Cohen AE (2016) Optical electrophysiology for probing function and pharmacology of voltage-gated ion channels. Elife 5:e15202. 